\title{
Photoluminescence probing of interface evolution with annealing in InGa(N)As/GaAs single quantum wells
}

\author{
Jun Shao, ${ }^{1, a)}$ Zhen Qi, ${ }^{1}$ H. Zhao, ${ }^{2}$ Liang Zhu, ${ }^{1}$ Yuxin Song, ${ }^{3}$ Xiren Chen, ${ }^{1}$ F.-X. Zha, ${ }^{4}$ \\ Shaoling Guo, ${ }^{1}$ and S. M. Wang ${ }^{2,3, b)}$ \\ ${ }^{1}$ National Laboratory for Infrared Physics, Shanghai Institute of Technical Physics, Chinese Academy \\ of Sciences, 200083 Shanghai, China \\ ${ }^{2}$ Department of Microtechnology and Nanoscience, Chalmers University of Technology, S-41296 Göteborg, \\ Sweden \\ ${ }^{3}$ State Key Laboratory of Functional Materials for Informatics, Shanghai Institute of Microsystem \\ and Information Technology, Chinese Academy of Sciences, 200050 Shanghai, China \\ ${ }^{4}$ Physics Department, Shanghai University, 200444 Shanghai, China
}

(Received 28 July 2015; accepted 10 October 2015; published online 23 October 2015)

\begin{abstract}
The effects of thermal annealing on the interfaces of $\mathrm{InGa}(\mathrm{N}) \mathrm{As} / \mathrm{GaAs}$ single quantum wells (SQWs) are investigated by excitation-, temperature-, and magnetic field-dependent photoluminescence (PL). The annealing at $750{ }^{\circ} \mathrm{C}$ results in more significant blueshift and narrowing to the PL peak than that at $600^{\circ} \mathrm{C}$. Each of the PL spectra can be reproduced with two PL components: (i) the low-energy component (LE) keeps energetically unchanged, while the high-energy component (HE) moves up with excitation and shows at higher energy for the $\operatorname{In}_{0.375} \mathrm{Ga}_{0.625} \mathrm{As} / \mathrm{GaAs}$ but crosses over with the LE at a medium excitation power for the $\operatorname{In}_{0.375} \mathrm{Ga}_{0.625} \mathrm{~N}_{0.012} \mathrm{As}_{0.988} / \mathrm{GaAs}$ SQWs. The HE is broader than the corresponding LE, the annealing at $750{ }^{\circ} \mathrm{C}$ narrows the $\mathrm{LE}$ and $\mathrm{HE}$ and shrinks their energetic separation; (ii) the PL components are excitonic, and the InGaNAs shows slightly enhanced excitonic effects relative to the InGaAs SQW; (iii) no typical S-shape evolution of PL energy with temperature is detectable, and similar blueshift and narrowing are identified for the same annealing. The phenomena are mainly from the interfacial processes. Annealing improves the intralayer quality, enhances the interfacial In-Ga interdiffusion, and reduces the interfacial fluctuation. The interfacial interdiffusion does not change obviously by the small $\mathrm{N}$ content and hence similar PL-component narrowing and blueshift are observed for the SQWs after a nominally identical annealing. Comparison with previous studies is made and the PL measurements under different conditions are shown to be effective for probing the interfacial evolution in QWs. (C) 2015 AIP Publishing LLC. [http://dx.doi.org/10.1063/1.4934523]
\end{abstract}

\section{INTRODUCTION}

Photoluminescence (PL) as a powerful optical spectroscopy has been widely applied in characterizing semiconductors and quantum well (QW) structures, ${ }^{1,2}$ of which the transition energy, intensity, and linewidth have been frequently employed for extracting physical properties. ${ }^{3-6}$ With improved spectral resolution and signal-to-noise ratio (SNR), curve fitting analysis of PL spectrum can be performed, which provides more details about the PL processes involved and the competition in between; ${ }^{7,8}$ using different experimental conditions of excitation-power, temperature and magnetic field, bandedge electronic structure, shallow impurity levels, and/or interfacial process can be identified. ${ }^{9,10}$

Dilute nitride $\operatorname{In}_{x} \mathrm{Ga}_{1-x} \mathrm{~N}_{y} \mathrm{As}_{1-y}$ has been extensively studied because of the large bandgap bowing and applications for GaAs-based telecom lasers. ${ }^{11-14}$ The optical quality deteriorates significantly with increasing $\mathrm{N}$ content due to the formation of deep levels with an associated $S$-shape evolution of transition energy in temperature-dependent PL measurements, and the $S$-shape diminishes with In content $x$ and vanishes when $x \gtrsim 0.3 .^{15,16}$ To improve the optical quality, either in

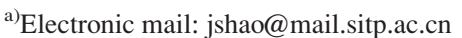

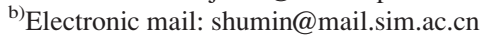

situ annealing or post-growth rapid thermal annealing (RTA) is necessary, at the expense of a wavelength blueshift. ${ }^{17}$ As $\mathrm{PL}$ is more sensitive to the change in composition profile than other methods, such as X-ray diffraction, ${ }^{18,19}$ it was frequently used for clarifying the blueshift. ${ }^{20,21}$ The evolution of PL peak energy manifested pronounced $S$-shape for the dilute-N InGaNAs/GaAs single quantum wells (SQWs) at low temperatures, and followed the empirical Varshni model in the high-temperature region with significant reduction in the temperature dependence as compared to the $\mathrm{N}$-free sample. ${ }^{22-25}$ However, (i) controversies remained that while the drastic blueshift after annealing was considered as a result of the $\mathrm{N}$ content and/or the N-related localized level in the QW layer but not the In-Ga interdiffusion, ${ }^{20,23,24}$ it was indicated that the annealing alone may induce interdiffusion in a length of $\leq 2 \mathrm{~nm} ;^{25}$ and (ii) while an exciton binding energy was derived to be about $6.5 \mathrm{meV}$ for $\mathrm{In}_{0.05} \mathrm{Ga}_{0.95} \mathrm{As} / \mathrm{GaAs} \mathrm{QW}$ with a 8-nm-thick well layer, ${ }^{26}$ a very small $\mathrm{N}$ content of about $0.5 \%$ in a $6-\mathrm{nm} \mathrm{In}_{0.4} \mathrm{Ga}_{0.6} \mathrm{~N}_{0.005} \mathrm{As}_{0.995} / \mathrm{GaAs} \mathrm{SQW}$ was shown to increase surprisingly the exciton binding energy by nearly $80 \%$ relative to the referential InGaAs/GaAs SQW to a value of about $17.5 \mathrm{meV}{ }^{27}$

In this work, the RTA effects on $\operatorname{InGa}(\mathrm{N}) \mathrm{As} / \mathrm{GaAs}$ SQWs are characterized by PL measurements at different 
excitations, temperatures, and magnetic fields, with the assistance of PL component analysis and bandedge electronic structure calculation. While no typical $S$-shape evolution of PL-peak energy with temperature is detectable, the PL peak blueshifts at similar levels for both the InGa(N)As SQWs after the same annealing. The PL spectra are well reproduced by a two-PL-component assumption, and the PL components are excitonic and of interband ground-state transition. Incorporation of dilute nitrogen into the $\mathrm{QW}$ layer moderately increases the exciton binding energy and reduced effective mass. Annealing improves interlayer crystal quality, enhances interfacial interdiffusion, and reduces the QW thickness and the interface fluctuation. Comparison suggests that PL measurements under different conditions with aid of curve fitting analysis may serve as an efficient vehicle for probing the interfacial evolution in QWs. The paper is organized as follows: In Sec. II, brief introduction is made to the PL experiments as well as the sample structures and RTA conditions. In Sec. III, excitation-power dependent PL analysis is first conducted, followed by magneto-PL analysis in Sec. III B, temperature-dependent investigation in Sec. III C, and mechanism discussion in Sec. IIID. A conclusion is given in Sec. IV.

\section{EXPERIMENT}

Two $\mathrm{In}_{x} \mathrm{Ga}_{1-x} \mathrm{As} / \mathrm{GaAs}$ (denoted by A hereafter) and $\mathrm{In}_{x} \mathrm{Ga}_{1-x} \mathrm{~N}_{y} \mathrm{As}_{1-\gamma} / \mathrm{GaAs}$ (denoted by B) SQW structures were grown on (100) GaAs substrate by an EPI930 molecular beam epitaxial system. The structure starts with a 200-nm

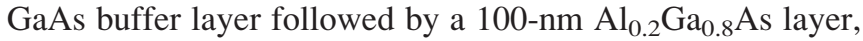
the active region consists of a 24 monolayer (ML) thick $\mathrm{InGa}(\mathrm{N}) \mathrm{As}$ QW sandwiched between two 100-nm GaAs barrier layers. Another $100-\mathrm{nm} \mathrm{Al}_{0.2} \mathrm{Ga}_{0.8} \mathrm{As}$ layer and a $10-\mathrm{nm}$ GaAs cap layer were grown above. The growth temperature was $580^{\circ} \mathrm{C}$ for the GaAs buffer and $\mathrm{AlGaAs}$ cladding layer, and $450^{\circ} \mathrm{C}$ for the QW layer. The growth rates of InAs and GaAs were 0.5 and $1.0 \mathrm{ML} / \mathrm{s}$, respectively. The In content $x$ was characterized by rocking curve analysis as 0.375 , and the thickness of the QW layer was about $7 \mathrm{~nm}$. The structure $\mathrm{B}$ was grown with nitrogen $(\mathrm{N})$ source open during the growth of QW layer, and the $\mathrm{N}$ content $y$ was derived as $1.2 \% .{ }^{17}$ Each of the structures was cut into three samples, of which one was taken as a reference (labeled by A/B-ag), and the other two were treated by post-growth rapid thermal annealing under 600 and $750^{\circ} \mathrm{C}$ for $30 \mathrm{~s}$ (by $\mathrm{A} / \mathrm{B}-600$ and A/B-750). More details can be found elsewhere. ${ }^{17}$

PL measurements were performed with a Fourier transform infrared spectrometer-based PL system. ${ }^{2}$ The spectrometer was equipped with an InGaAs photodiode detector and worked in the continuous-scan rather than step-scan mode. ${ }^{8,28}$ $\mathrm{An} \mathrm{Ar}^{+}$-ion laser configured at $514.5 \mathrm{~nm}$ line was used for pumping, and the laser beam was focused on the sample with a diameter of about $0.5 \mathrm{~mm}$, the power was exactly adjusted with a laser power controller in a range of 2-128 $\mathrm{mW}$ (equivalently, a power density range of $1-64 \mathrm{~W} / \mathrm{cm}^{2}$ ) for the excitation power-dependent PL measurements. A spectral resolution of 0.5 or $1.0 \mathrm{meV}$ was set for the temperature/excitation-power or magnetic-field dependent measurements. A close-cycle cryostat was used for the samples' temperatures of $11-300 \mathrm{~K}$, its position was controlled to warrant the PL intensity a comparable quantity. A magneto-optical superconducting magnet in Faraday configuration ${ }^{10,29}$ was employed for the magneto-PL measurements at $4.5 \mathrm{~K}$.

\section{RESULTS AND DISCUSSION}

Figure 1 illustrates the representative PL spectra of the InGaAs (A-ag, A-600 and A-750) and InGaNAs (B-ag, B-600 and B-750) SQWs at $11 \mathrm{~K}$. Also plotted are the curve fittings of the spectra in dots, dashed-dots, and dashes.

The PL spectra show asymmetric lineshapes, and each can be reproduced by two PL components with Gaussian profile. Incorporation of nitrogen leads to an obvious redshift of the PL peak. The $600^{\circ} \mathrm{C}$ annealing reduces the peak intensity and broadens the linewidth of the InGaAs QW sample, while the $750{ }^{\circ} \mathrm{C}$ annealing shrinks the linewidth and enhances obviously the intensity of the InGaNAs sample. The PL peak shows up at 1.134 and $1.081 \mathrm{eV}$ for the as-grown A-ag and $\mathrm{B}-\mathrm{ag}$, moves to 1.141 and $1.097 \mathrm{eV}$ for the annealed A-600 and B-600, and 1.174 and $1.132 \mathrm{eV}$ for the A-750 and B-750 samples. The $750{ }^{\circ} \mathrm{C}$ annealing induces an abrupt blueshift of the PL peak relative to that the $600^{\circ} \mathrm{C}$, and reduces the energy difference from $53 \mathrm{meV}$ between the A-ag and B-ag to $42 \mathrm{meV}$ between the A-750 and B-750 samples.

These phenomena reflect the $\mathrm{N}$-induced effects in the InGaNAs and the influences of the RTA in the InGaAs and InGaNAs SQWs. To clarify the mechanisms behind, excitation-power, magnetic-field, and temperature dependent PL analyses are conducted.

\section{A. Excitation-power dependent PL: Energy cross-over and linewidth broadening of two PL components}

Excitation-power dependent PL measurements were carried out at $11 \mathrm{~K}$. The representative PL spectra are depicted in Fig. 2 for the as-grown InGaAs (a) and InGaNAs (b)

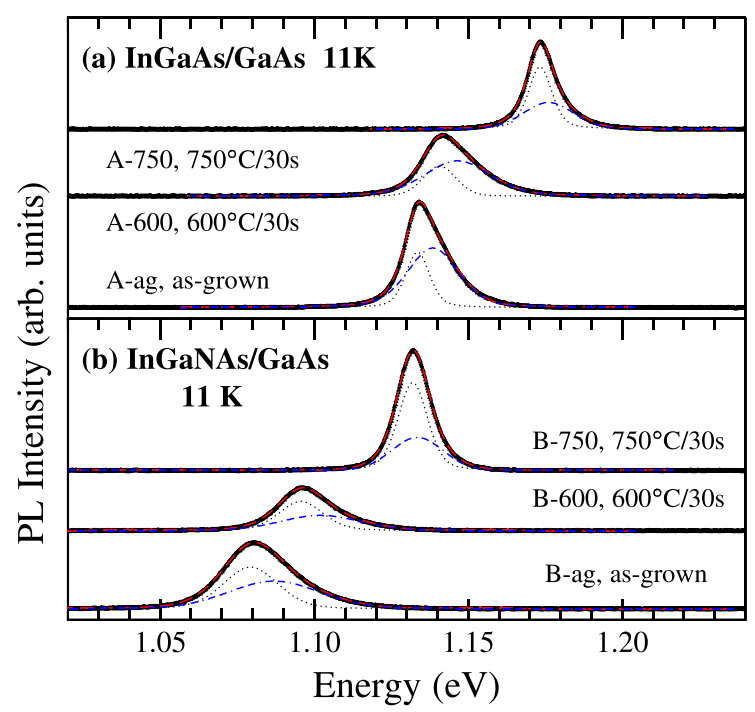

FIG. 1. PL spectra of (a) InGaAs/GaAs (A-ag, A-600, and A-750) and (b) InGaNAs/GaAs (B-ag, B-600, and B-750) SQWs at $11 \mathrm{~K}$. Dots, dashed-dots, and dashes for curve fittings. 


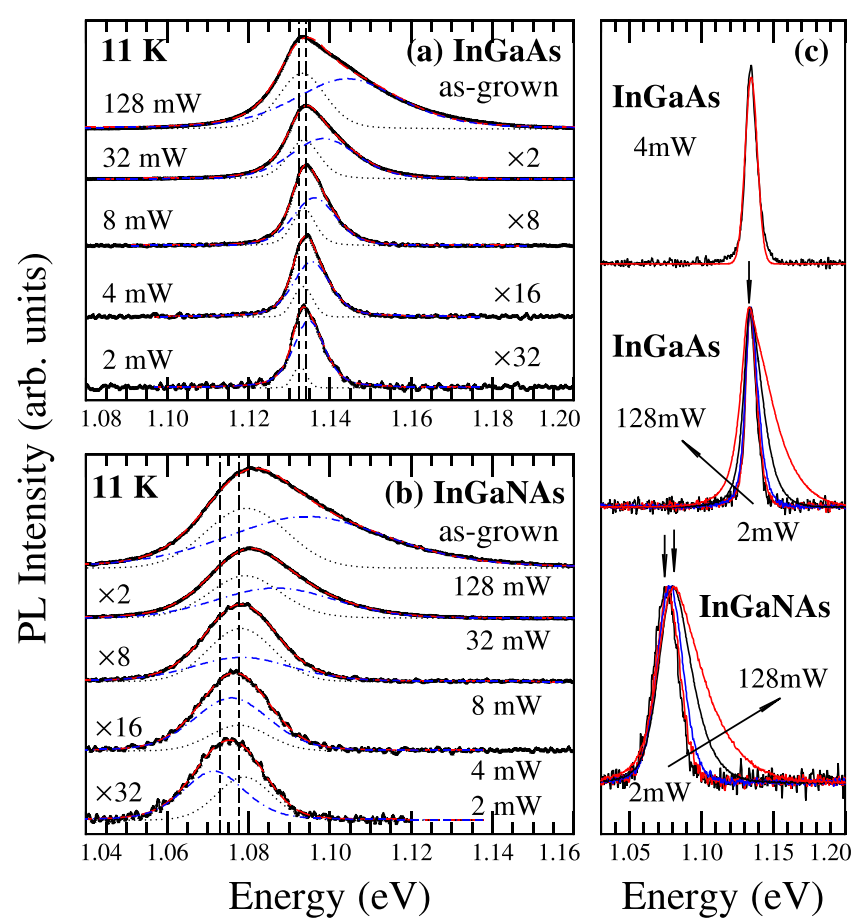

FIG. 2. Excitation power-dependent PL spectra of as-grown InGaAs/GaAs (a) and InGaNAs/GaAs (b) SQWs at $11 \mathrm{~K}$, magnified to a similar peak height. Dots and dashed-dots for curve fittings, vertical dashes for PLcomponent peaks at $2 \mathrm{~mW}$ excitation. (c) The spectra are normalized and overlaid for the SQWs. Vertical arrows for main PL peak positions.

SQWs, respectively. The PL spectra are magnified for clear line shapes at lower excitation.

The linewidth of the InGaAs is significantly narrower than that of the InGaNAs, and both broaden as the excitation gets enhanced. The PL spectra are asymmetric, and can be fitted by two PL components with Gaussian profile as plotted in dots, dashed-dots, and dashes. The vertical dashes mark the energetic positions of the two PL components at an excitation of $2 \mathrm{~mW}$. While one PL component keeps energetically unchanged (denoted with standing- $P L$ component), the other moves to higher energies (denoted as moving- $P L$ component) as the excitation power gets stronger. For the InGaAs SQW, the moving-PL component shows up on the high-energy side of the standing-PL component; for the InGaNAs SQW, however, the moving-PL component shows up on the low-energy side at low excitation, crosses over with the standing- $P L$ component at about $8 \mathrm{~mW}$, and moves up to the high-energy side as the excitation gets stronger further. To verify the rationality of the fittings, the PL spectra are normalized and overlaid in Fig. 2(c) for the as-grown InGaAs and InGaNAs samples, respectively. It is obvious that (i) even the rather symmetric PL spectrum of the InGaAs sample at $4 \mathrm{~mW}$ could not be fitted by single component satisfactorily; (ii) while the high-energy edge of the PL spectrum blueshifts significantly as the excitation power increases from 2 to $128 \mathrm{~mW}$ for the both samples, the lowenergy edge of the PL spectrum redshifts slightly for the InGaAs sample but keeps nearly unchanged for the InGaNAs sample; and (iii) while the main PL peak position of the InGaAs sample does nearly not change with excitation power, that of the InGaNAs sample moves slightly and gradually as the excitation rises up from 2 to $8 \mathrm{~mW}$, but jumps to an obviously higher energy for the high excitation of 32 and $128 \mathrm{~mW}$, as marked by the vertical arrows. This observation approves the two-component assumption, the different evolutions of the two components' positions for the two samples at relatively low excitation as well as a crossover for the InGaNAs sample between 8 and $32 \mathrm{~mW}$.

To reveal the evolution of the two PL components' energies with excitation power, a plot is drawn in Fig. 3 for the as-grown and annealed InGaAs (a) and InGaNAs (b) SQWs. The solid squares and open triangles represent the standingand moving- $P L$ components, while the dashes and solid lines serve as guides to the eyes. The data and the guide lines are shifted $10 \mathrm{meV}$ downward for the as-grown samples for clarity.

The energy of the standing- $P L$ component keeps nearly unchanged with the excitation power, while that of the moving- $L$ component shifts to higher energies monotonically and keeps at high-energy side in the whole excitation power range for the InGaAs SQWs. For the InGaNAs SQWs, however, there is a cross-over between the two PL components at about $8 \mathrm{~mW}$. The blueshift of the moving component manifests nearly linear characteristic against the logarithm of the excitation power, $\ln \mathrm{P}$. The energetic separation between the two PL components is about 11, 9, and $7 \mathrm{meV}$ for the A-ag, A-600, and A-750 InGaAs, and 15, 16, and $7 \mathrm{meV}$ for the B-ag, B-600, and B-750 InGaNAs SQWs at $128 \mathrm{~mW}$, respectively.

The linewidth as a function of excitation power is depicted in Fig. 4 for the two PL components of the as-grown (a), $600^{\circ} \mathrm{C}$ annealed (b), and $750^{\circ} \mathrm{C}$ annealed (c) InGaAs (circles and open triangles) and InGaNAs (squares and filled triangles) SQWs. The dashes and solid lines serve as guides to the eyes. The scale of the vertical axis is set to an identical range of $0-70 \mathrm{meV}$. The values for the extremely low and

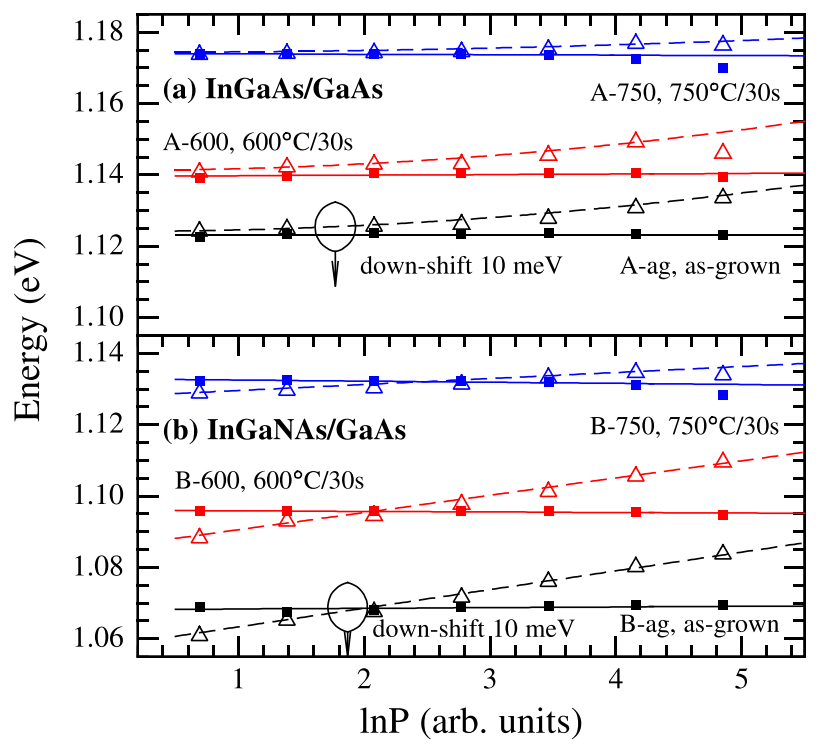

FIG. 3. Energy vs. excitation power for the PL components of as-grown and annealed InGaAs/GaAs (a) and InGaNAs/GaAs (b) SQWs at $11 \mathrm{~K}$. Solid squares and open triangles for standing- and moving- $P L$ components, 10-meV downshift for as-grown samples for clarity, dashes and solid lines for guiding the eyes. 


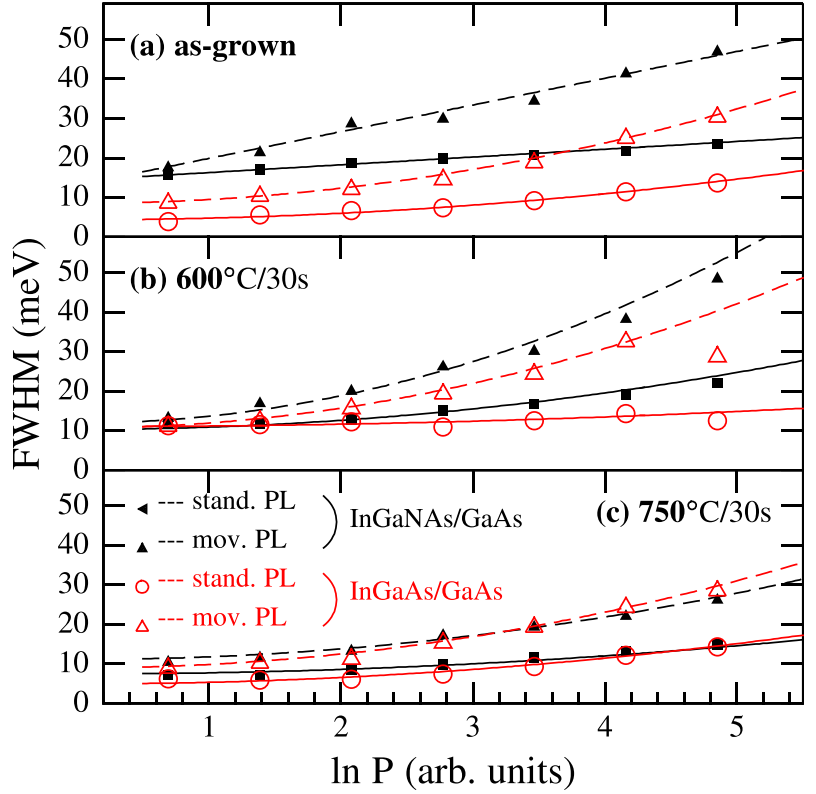

FIG. 4. Linewidth vs. excitation power for the two PL components of asgrown and annealed InGaAs/GaAs (circles and open triangles) and InGaNAs/GaAs (squares and filled triangles) SQWs at $11 \mathrm{~K}$. Dashes and solid lines guide the eyes.

high excitation ( 2 and $128 \mathrm{~mW}$ ) are listed in Table I: at 2 $\mathrm{mW}$, the linewidths of the standing- and moving-PL components are 4 and $9 \mathrm{meV}, 11$ and $12 \mathrm{meV}$, and 6 and $10 \mathrm{meV}$ for the A-ag, A-600, and A-750 InGaAs; and 16 and $18 \mathrm{meV}, 12$ and $14 \mathrm{meV}$, and 7 and $11 \mathrm{meV}$ for the B-ag, B-600, and B750 InGaNAs SQWs, respectively. At $128 \mathrm{~mW}$, the corresponding values get to 14 and $31 \mathrm{meV}, 13$ and $30 \mathrm{meV}$, and 14 and $29 \mathrm{meV}$ for the A-ag, A-600, and A-750 InGaAs; and 24 and $48 \mathrm{meV}, 22$ and $49 \mathrm{meV}$, and 15 and $27 \mathrm{meV}$ for the B-ag, B-600, and B-750 InGaNAs SQWs.

The phenomena indicate that (i) the PL lineshape is not dominated by below-gap bandtail states for the InGaAs SQW, as the moving-PL component shows up at high-energy side even at low excitation power; the annealing does not improve the PL intensity, and the annealing-induced blueshift is hence not the result of diminishing the bandtails as previously considered. ${ }^{17}$ (ii) The cross-over of the two PL components for the InGaNAs SQW does not favor the bandtail assumption, and the annealing-induced narrowing and reduction of the two components' separation seem to be

TABLE I. FWHM of the standing- and moving- $P L$ components for the InGaAs/GaAs (A-ag, A-600, and A-750) and InGaNAs/GaAs (B-ag, B-600, and B-750) SQW samples at $11 \mathrm{~K}$ with excitation power of 2 and $128 \mathrm{~mW}$, respectively.

\begin{tabular}{lcccc}
\hline \hline Sample & $\begin{array}{c}\text { FWHM } \\
\text { standing-PL }\end{array}$ & $\begin{array}{r}\text { (@2 mW) } \\
\text { moving-PL }\end{array}$ & $\begin{array}{c}\text { FWHM } \\
\text { standing-PL }\end{array}$ & $\begin{array}{c}\text { (@128 mW) } \\
\text { moving-PL }\end{array}$ \\
\hline A-ag & 4 & 9 & 14 & 31 \\
A-600 & 11 & 12 & 13 & 30 \\
A-750 & 6 & 10 & 14 & 29 \\
B-ag & 16 & 18 & 24 & 48 \\
B-600 & 12 & 14 & 22 & 49 \\
B-750 & 7 & 11 & 15 & 27 \\
\hline \hline
\end{tabular}

inconsistent with the consideration of In-Ga interdiffusion and local nitrogen environment reorganization. ${ }^{30}$ (iii) No Mott transition is seen even with the highest excitation power, ${ }^{26}$ indicating the low-excitation characteristic of this measurement and the non-free-carrier transition of the moving-PL component.

For further insight in the moving- and standing- $P L$ components, magneto-PL analysis is carried out.

\section{B. Magneto-PL: Excitonic effect}

Magneto-PL measurements were performed in a field range of $0-10 \mathrm{~T}$ at $4.5 \mathrm{~K}$, with the magnetic field being perpendicular to the sample's surface. The excitation power was set at $80 \mathrm{~mW}$ to warrant the moving-PL component showing up on the high-energy side of the standing- $P L$ component. The representative spectra are depicted in Fig. 5 for the $600{ }^{\circ} \mathrm{C}$ annealed InGaAs/GaAs (a) and InGaNAs/GaAs (b) SQW samples, respectively. Vertical dashes indicate the main PL peak at $B=0 \mathrm{~T}$, the main PL peak blueshifts obviously as the magnetic field rises, especially for the InGaAs SQW sample. The PL spectra show obvious asymmetric lineshape, and the two-component assumption applies for curve fittings. The results are plotted in dots and dashed-dots for the standing- and moving- $P L$ components with Gaussian profile. The PL intensity is enhanced as the field gets higher especially for the InGaAs SQW and the moving- $P L$ components, as depicted in Figs. 5(c) and 5(d) for the integral intensities of the standing- $P L(\mathrm{sp})$, moving- $P L(\mathrm{mp})$, and their summation $(\mathrm{sp}+\mathrm{mp})$ of the InGaAs and InGaNAs SQW samples, respectively. Similar magnetic field-induced enhancement of oscillator strength (and hence integral intensity) was observed previously in InGaAs/InP quantum wells,

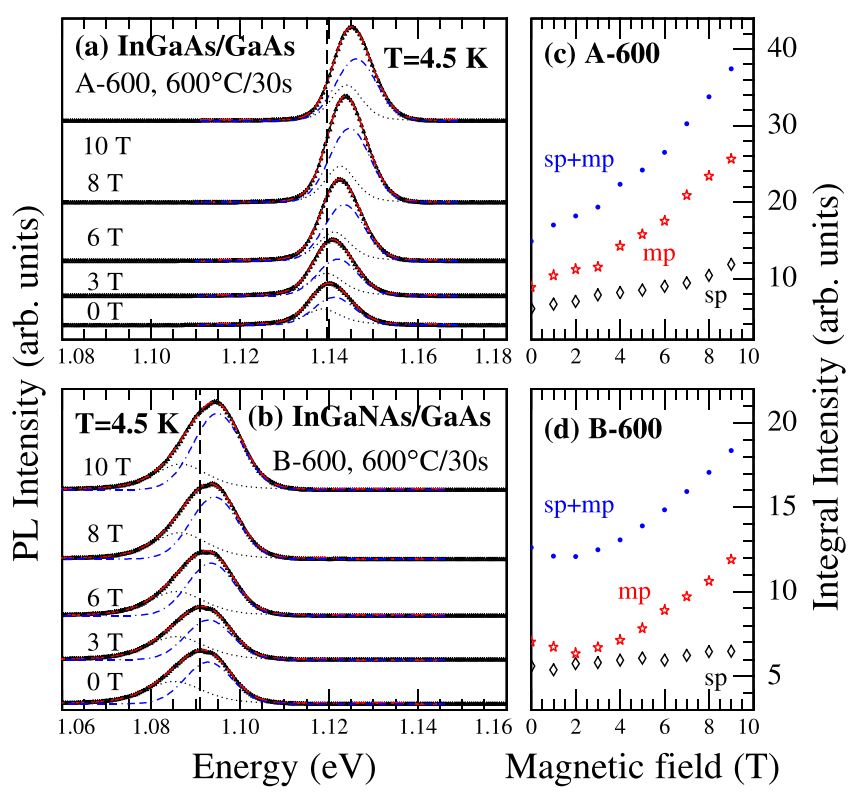

FIG. 5. Magneto-PL spectra of InGaAs/GaAs A-600 (a), InGaNAs/GaAs B600 (b), and corresponding integral insensities (c) and (d) at $4.5 \mathrm{~K}$. Dots and dashed-dots in (a) and (b) for curve fittings, vertical dashes for peak position at $0 \mathrm{~T}$. $\mathrm{sp}, \mathrm{mp}$, and $\mathrm{sp}+\mathrm{mp}$ in (c) and (d) for stand-, moving-PL, and their summation. 
and was shown to be due to the shrinkage of quasi-twodimensional exciton wave functions. ${ }^{31,32}$

Figure 6 plots the energies of the two PL components and the whole PL peak against magnetic field in squares, triangles, and open circles for the A-600 (a) and B-600 (b) SQWs. Similar to the PL peak, the two PL components manifest definitely blueshift with magnetic field, and the blueshift is relatively stronger for the A-600 SQW.

From theoretical viewpoint, the relative motion of the electron and hole constituting an exciton in QW is deformed by Lorentz forces when an external magnetic field $B$ is applied to the exciton. For the exciton ground state with an S-like envelope wavefunction, the deformation leads to admixture with P-like envelope states. The resultant angular momentum is proportional to and antiparallel with $B$, the magnetic dipole's energy is also proportional to $B .{ }^{33}$ The excitonic transition energy will therefore undergo a quadratic blueshift with $B$, which is known as diamagnetic shift and can be described quantitatively by a quasi-two-dimensional magneto-exciton model $^{29}$

$$
\begin{gathered}
\left\{-\frac{\hbar^{2}}{2 \mu}\left[\frac{1}{\rho} \frac{\partial}{\partial \rho}\left(\rho \frac{\partial}{\partial \rho}\right)\right]+\frac{e^{2}}{8 \mu} B^{2} \rho^{2}+V(\rho)\right\} R(\rho)=E R(\rho), \\
V(\rho)=\frac{-e^{2}}{4 \pi \varepsilon_{0}} \iint_{-\infty}^{+\infty} \frac{\left|Z_{e}\left(z_{e}\right)\right|^{2}\left|Z_{h}\left(z_{h}\right)\right|^{2}}{\varepsilon_{r}\left(z_{e}, z_{h}\right) \sqrt{\rho^{2}+\left(z_{e}-z_{h}\right)^{2}}} d z_{e} d z_{h},
\end{gathered}
$$

where $\rho$ is the in-well-plane separation between the electron and hole constituting an exciton. $\mu$ is the exciton reduced effective mass. $Z_{e}\left(z_{e}\right)$ and $Z_{h}\left(z_{h}\right)$ are the confined-state wavefunctions of electrons and holes, and are calculated by a model-solid method. ${ }^{34-36} \varepsilon_{r} \varepsilon_{0}$ is the dielectric constant of the well or the barrier depending on $z_{e}$ and $z_{h}$. $R(\rho)$ is excitonic envelope function. The theoretical excitonic energy shift is plotted in Fig. 6 as solid line and dashed-dots for the main

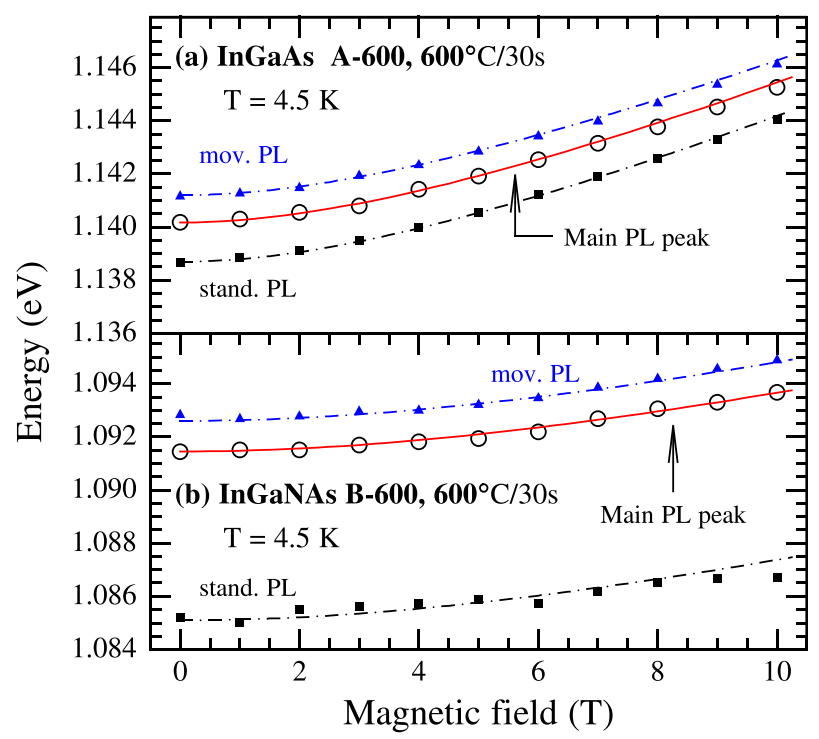

FIG. 6. Energy vs. magnetic field for (a) InGaAs/GaAs A-600 and (b) InGaNAs/GaAs B-600 SQWs at 4.5 K. Lines and dashed-dots for theoretical excitonic diamagnetic shift.
PL peak and its two PL components, the good agreements with the experimental data indicate the excitonic nature of the two PL components. This is indeed consistent with the magnetic-field induced enhancement of the integral intensities as illustrated in Figs. 5(c) and 5(d). The exciton reduced effective mass and binding energy are derived as listed in Table II.

The InGaAs SQW samples have smaller $E_{b}$ and $\mu$ than the corresponding InGaNAs counterparts, the $600^{\circ} \mathrm{C}$ annealing does not change the $E_{b}$ and $\mu$, while the $750^{\circ} \mathrm{C}$ reduces the values significantly. For the InGaNAs SQW, the $600^{\circ} \mathrm{C}$ annealing drastically increases the $E_{b}$ and $\mu$, while the $750{ }^{\circ} \mathrm{C}$ reduces the values slightly relative to the as-grown sample. The as-grown and $600^{\circ} \mathrm{C}$ annealed samples have slightly larger, while the $750^{\circ} \mathrm{C}$ samples have smaller $E_{b}$ and $\mu$ for the moving- $P L$ components. These indicate that (i) the standing- and moving-PL components are not bandtail- and/ or localization-related, but of similar interband exciton, due to the very similar excitonic parameters and the annealingdependent energetic separation between the two components; (ii) annealing introduces different effects on the InGaAs and InGaNAs SQWs; and (iii) the introduction of $1.2 \%$ nitrogen into InGaAs leads to an increased exciton binding energy by about $18 \%, 38 \%$, and $15 \%$, respectively, for the as-grown, $600{ }^{\circ} \mathrm{C}$, and $750{ }^{\circ} \mathrm{C}$ annealed samples.

To trace out the real origin of the excitonic nature and the annealing effects, lineshape analysis of the PL spectra in a wide temperature range is conducted.

\section{Temperature-dependent PL: Electron-phonon coupling}

Temperature-dependent PL measurements were conducted with an excitation power of $32 \mathrm{~mW}$ to warrant the moving- $P L$ component showing up on the high-energy side of the standing-PL component. The representative PL spectra are plotted in Fig. 7 for the as-grown InGaAs (a) and InGaNAs (b) SQW samples in 11-290 K, respectively. The spectra of $150 \mathrm{~K}$ and above are magnified for tracing the PL peaks. The vertical dashed-dots mark the main PL peaks at $11 \mathrm{~K}$, by which the redshift of PL peak is obvious for the temperatures above $40 \mathrm{~K}$.

The PL spectra can be well fitted with the aforementioned two-component assumption as shown by dashes for the spectra taken at $11 \mathrm{~K}$, by which the lineshape parameters of integral intensity, energy, and FWHM are obtained for

TABLE II. Exciton binding energy $E_{b}$ in meV and reduced effective mass $\mu$ in $m_{e}$ deduced from magneto-PL for InGaAs/GaAs (A-ag, A-600, and A-750) and InGaNAs/GaAs (B-ag, B-600, and B-750) SQW samples.

\begin{tabular}{lcccccc}
\hline \hline Sample & $\begin{array}{c}\text { Main } \\
E_{b}\end{array}$ & $\begin{array}{c}\text { Peak } \\
\mu\end{array}$ & $\begin{array}{c}\text { Standing } \\
E_{b}^{s}\end{array}$ & $\begin{array}{c}\text { PL } \\
\mu^{s}\end{array}$ & $\begin{array}{c}\text { Moving } \\
E_{b}^{m}\end{array}$ & $\begin{array}{c}\text { PL } \\
\mu^{m}\end{array}$ \\
\hline A-ag & 8.2 & 0.045 & 7.8 & 0.042 & 8.2 & 0.045 \\
A-600 & 8.2 & 0.045 & 8.1 & 0.044 & 8.4 & 0.046 \\
A-750 & 7.6 & 0.040 & 7.8 & 0.042 & 7.7 & 0.041 \\
B-ag & 9.7 & 0.056 & 9.6 & 0.055 & 9.8 & 0.057 \\
B-600 & 11.3 & 0.071 & 11.2 & 0.070 & 11.3 & 0.07 \\
B-750 & 8.7 & 0.048 & 9.3 & 0.053 & 8.4 & 0.046 \\
\hline \hline
\end{tabular}




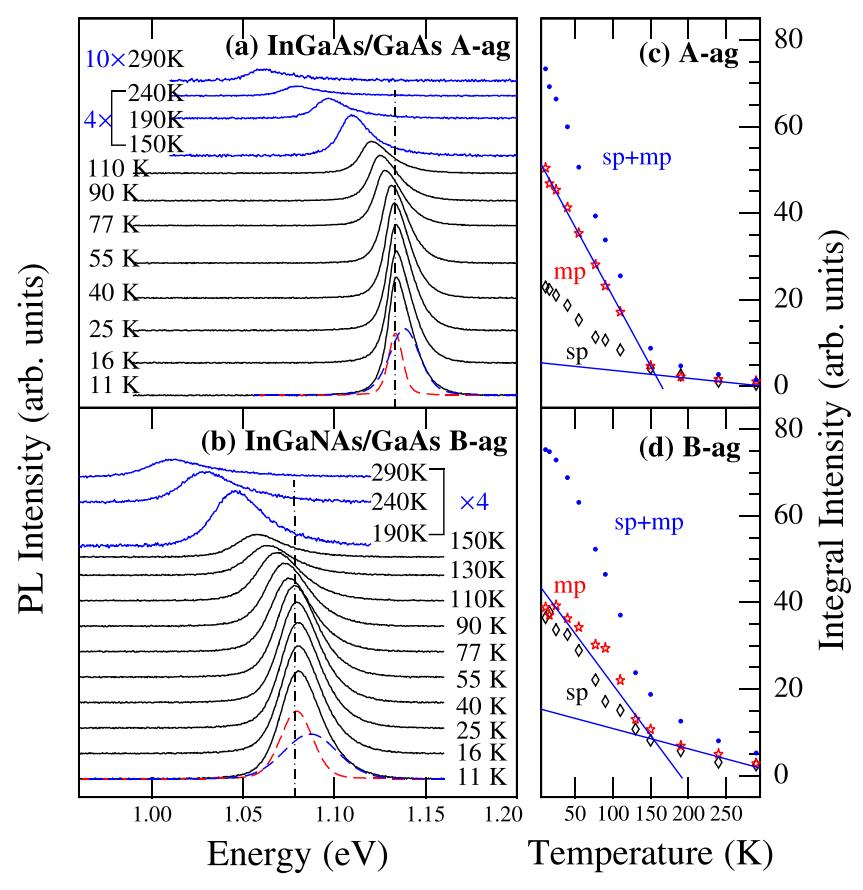

FIG. 7. PL spectra of as-grown InGaAs/GaAs (a), InGaNAs/GaAs (b), and corresponding integral intensities (c) and (d) in a temperature range of 11-290 K. Vertical dashed-dots in (a) and (b) mark peak position at $11 \mathrm{~K}$, solid lines in (c) and (d) represent slopes of integral intensity vs. temperature.

each of the PL components, and the integral intensities of the standing ( $\mathrm{sp}$ ) and moving (mp) components as well as their summation $(\mathrm{sp}+\mathrm{mp})$ are plotted in Figs. 7(c) and 7(d) for the InGaAs and InGaNAs SQW samples, respectively. It is worthy to mention that though at low temperatures, the two components are both of the Gaussian profile, a mixture of Gaussian and Lorentzian is better for the moving-component in the temperature range above about $150 \mathrm{~K}$, and finally a pure Lorentzian profile is necessary at $290 \mathrm{~K}$. While inhomogeneous broadening has a Gaussian profile, homogeneous broadening has a Lorentzian profile, and LO-phonon scattering may dominate the broadening especially at higher temperatures. $^{37,38}$ The temperature evolution of the integral intensity as shown in Figs. 7(c) and 7(d) is in fact consistent with the change of the lineshape that its quenching with temperature manifests a turning point at around $150 \mathrm{~K}$ as indicated by the slopes of the two parts of the integral intensity versus temperature.

Figure 8 depicts the energies of the main PL-peak, standing-, and moving-PL components as crosses, squares, and open triangles, respectively, for the InGaAs and InGaNAs SQWs. The data of the as-grown samples are shifted $20 \mathrm{meV}$ downward for clarity.

The redshift with increasing temperature is the result of the band gap shrinkage, the latter is due primarily to the increase in the interatomic spacing and secondarily to the enhancement of carrier-phonon interaction as temperature increases. It can be quantitatively mimicked by an empirical formula as Varshni relation ${ }^{3}$

$$
E_{i}(T)=E_{i}^{0}-\frac{\alpha_{i} T^{2}}{T+\beta_{i}},
$$

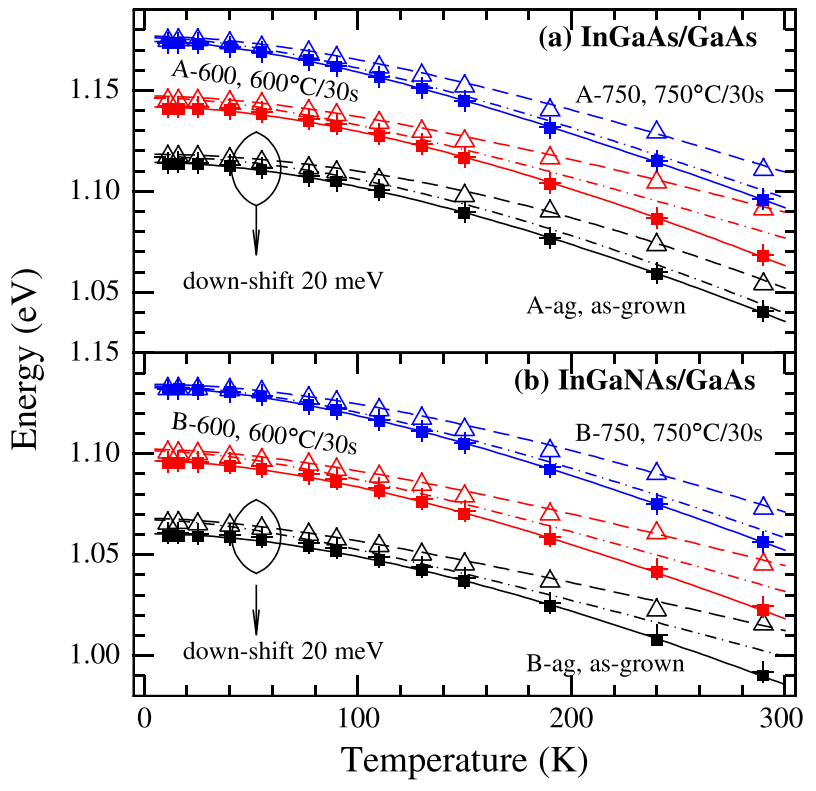

FIG. 8. Energy vs. temperature for the PL components of (a) InGaAs/GaAs and (b) InGaNAs/GaAs SQWs. Squares, open triangles, and crosses for standing-PL, moving-PL, and main PL peaks; dashes, solid lines, and dots for fittings by Varshni relation.

where $i$ is for the main peak (p), standing- (s), and moving$P L(\mathrm{~m})$, respectively. $E_{i}^{0}$ is the transition energy extrapolated to $0 \mathrm{~K}, \alpha_{i}$, and $\beta_{i}$ are fitting parameters of a particular PL transition. ${ }^{3}$ The results are overlaid directly on the experimental data as dots, solid line (coincident with the dots), and dashes, respectively, for the main PL peak, standing-, and moving-PL components. The deduced parameters are listed in Table III for the standing- $P L$, moving- $P L$, and main PL peaks. To check if the evolution of the moving-PL component contains band/level thermal filling effects, additional fitting is made to the moving- $P L$ component being subtracted by $k T$, and the result is depicted as dashed-dots in Fig. 8.

The main PL peak manifests similar characteristics of $\alpha_{i}$ and $\beta_{i}$ as the corresponding standing- $P L$ component for all the samples, the moving- $P L$, however, is rather different. The separation between the two PL components extrapolated to $0 \mathrm{~K}$ depends on annealing and has the smallest value of 2.6 and $1.7 \mathrm{meV}$ for the $750^{\circ} \mathrm{C}$ annealed InGaAs and InGaNAs SQW samples. The $750^{\circ} \mathrm{C}$ annealed samples have also the smallest $\alpha_{i}$ and $\beta_{i}$ for the main peak and standing-PL

TABLE III. Parameters of Varshni relation [Eq. (2)] for standing-PL (s), moving-PL (m), and main peak (p) energies of $\operatorname{InGaAs} / \mathrm{GaAs}$ (A) and InGaNAs/GaAs (B) SQWs. $E_{p}^{0}$ and $\Delta E$ in meV for main peak energy and energy difference between the two PL components at $0 \mathrm{~K}$. $\alpha$ in $\mathrm{meV} / \mathrm{K}$ and $\beta$ in $\mathrm{K}$.

\begin{tabular}{lcccccccc}
\hline \hline Sample & $E_{p}^{0}$ & $\begin{array}{c}\text { Main } \\
\alpha_{p}\end{array}$ & $\begin{array}{c}\text { Peak } \\
\beta_{p}\end{array}$ & $\begin{array}{c}\text { Standing } \\
\alpha_{s}\end{array}$ & $\begin{array}{c}\text { PL } \\
\beta_{s}\end{array}$ & $\begin{array}{c}\text { Moving } \\
\alpha_{m}\end{array}$ & $\begin{array}{c}\text { PL } \\
\beta_{m}\end{array}$ & $\Delta E$ \\
\hline A-ag & 1135.1 & 0.59 & 374 & 0.63 & 415 & 1.17 & 1277 & 4.1 \\
A-600 & 1142.9 & 0.61 & 391 & 0.62 & 406 & 0.34 & 229 & 5.4 \\
A-750 & 1174.4 & 0.46 & 205 & 0.46 & 201 & 0.42 & 254 & 2.6 \\
B-ag & 1081.6 & 0.66 & 498 & 0.62 & 448 & 0.28 & 147 & 7.6 \\
B-600 & 1097.2 & 0.55 & 343 & 0.54 & 318 & 0.32 & 195 & 5.9 \\
B-750 & 1133.0 & 0.52 & 281 & 0.50 & 260 & 0.49 & 391 & 1.7 \\
\hline \hline
\end{tabular}


component, and correspondingly, the largest main-peak redshifts of 78.2 and $76.4 \mathrm{meV}$ for the InGaAs and InGaNAs SQWs, respectively, as the temperature changes from 11 to $290 \mathrm{~K}$, relative to those of 74.1 and $72.5 \mathrm{meV}$ for the $600^{\circ} \mathrm{C}$ annealed and 74.1 and $69.5 \mathrm{meV}$ for the as-grown samples. For the InGaAs SQW, the moving- $P L$ manifests similar evolution of energy with temperature as the standing- $P L$ for the as-grown and $750^{\circ} \mathrm{C}$ annealed samples but weaker redshift than the standing- $P L$ for the $600{ }^{\circ} \mathrm{C}$ annealed sample, if thermal filling effect is excluded from the moving-PL component; for the InGaNAs SQW, however, the moving- $P L$ manifests weaker redshift than the standing- $P L$ also for the as-grown sample.

Figure 9 illustrates the evolution of the FWHM with temperature. The open circles and triangles represent the standing- and moving-PL components of the InGaAs, while the solid squares and triangles for those of the InGaNAs SQWs. The moving-PL component shows broader FWHM than the corresponding standing- $P L$, and both broaden as temperature rises. The FWHMs of the as-grown InGaNAs are broader than the corresponding ones of the InGaAs $\mathrm{SQW}$, the annealing reduces and that of $750{ }^{\circ} \mathrm{C}$ even eliminates the differences. For the InGaNAs SQW, annealing narrows the two PL components monotonically with raising the annealing temperature, while for the InGaAs SQW, $600^{\circ} \mathrm{C}$ annealing even slightly broadens the PL components.

The broadening with temperature indicates phonon interaction and can be described by the phenomenological expression for electron-phonon interaction based on the Bose-Einstein statistical factor ${ }^{38-40}$

$$
\Gamma_{i}(T)=\Gamma_{i}^{0}+\frac{\beta_{i}}{e^{\left\langle\hbar \omega_{L O}\right\rangle / k T}-1},
$$

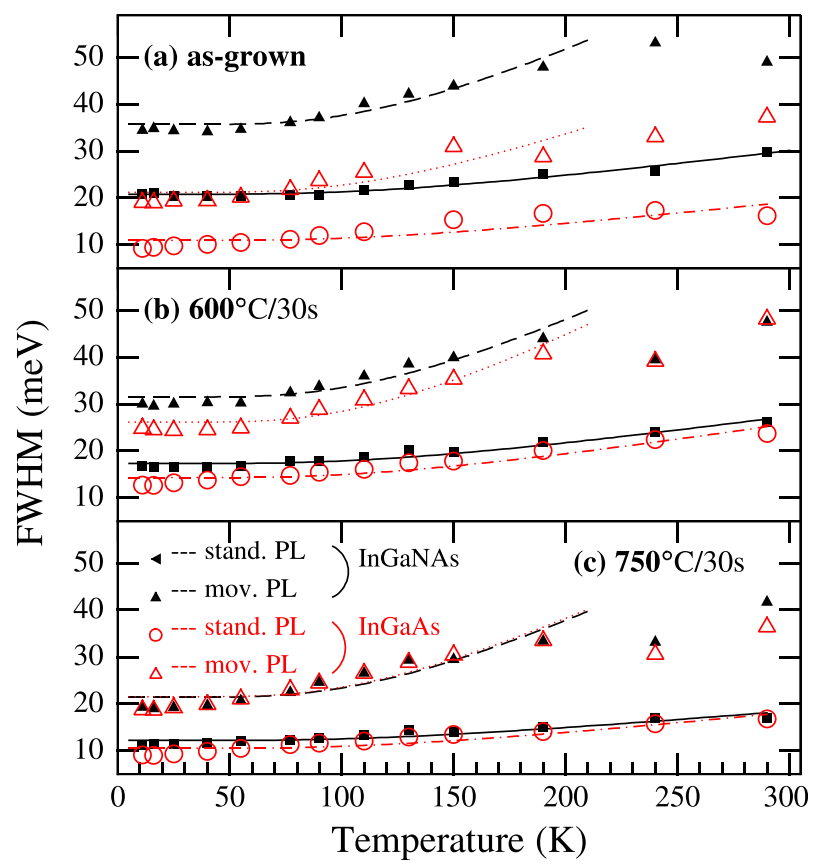

FIG. 9. FWHM vs. temperature for as-grown (a), $600^{\circ} \mathrm{C}$ (b), and $750^{\circ} \mathrm{C}$ (c) annealed SQWs. Open circles and triangles for standing- and moving- $P L$ of InGaAs/GaAs; solid squares and triangles for those of InGaNAs/GaAs SQWs. where $i$ is for the standing- (s) or moving-PL (m) component, $\Gamma_{i}^{0}$ is the phonon independent part of $\Gamma_{i}$ and related to the scattering with impurities and bound excitons. The second term arises from exciton scattering with optical phonons and $\beta_{i}$ for the scattering strengths, $\hbar \omega_{L O}$ is the LO phonon energy of the QW layer and taken as a linear interpolation of the values 36.6, 29.6, 92.1, and $74.4 \mathrm{meV}$ for GaAs, InAs, GaN, and $\mathrm{InN}^{.41,42}$

The results for the standing- and moving-PL components are overlaid directly on the corresponding experimental data as solid lines and dashes for the InGaNAs, and dashed-dots and dots for the InGaAs SQWs, respectively. The fitting parameters are listed in Table IV. For the standing- $P L$ component, the $\Gamma_{\mathrm{s}}^{0}$ is $10.5-14.3 \mathrm{meV}$ for the InGaAs, and $12.2-20.8 \mathrm{meV}$ for the InGaNAs SQWs; annealing from $600^{\circ} \mathrm{C}$ to $750^{\circ} \mathrm{C}$ first slightly increases and then reduces the value for the InGaAs but reduces monotonically significantly for the InGaNAs SQWs. The $\beta_{\mathrm{s}}$ is in a similar range of around $20 \mathrm{meV}$, and shows similar annealing dependence of first increase and then decrease for the two SQWs. For the moving- $P L$, the $\Gamma_{\mathrm{m}}^{0}$ is about double as the corresponding standing- $P L$ component, and also first increases and then turns around as the annealing changes from $600^{\circ} \mathrm{C}$ to $750^{\circ} \mathrm{C}$. The $\beta_{\mathrm{m}}$ is even about quadruple as that of the standing-PL component. While the as-grown InGaNAs SQW shows obviously larger $\Gamma_{\mathrm{i}}^{0}$ and $\beta_{\mathrm{i}}$ values than the InGaAs SQW, the annealing of $750^{\circ} \mathrm{C}$ significantly reduces and leads to similar values as the corresponding InGaAs SQW.

The evolution of energy and FWHM indicates that (i) both the standing- and moving-PL components originate in the inter-QW layer interband transition; the incorporation of $\mathrm{N}$ deteriorates the QW quality and broadens the FWHM, while the $750^{\circ} \mathrm{C}$ annealing improves the quality and makes it comparable to the InGaAs SQW. (ii) The standing- $P L$ component manifests similar redshift with temperature, while the moving- $P L$ shows weaker redshift even when thermal filling effect is considered for the as-grown and $600^{\circ} \mathrm{C}$ annealed, but similar redshift for the $750^{\circ} \mathrm{C}$ annealed SQWs when the thermal filling effect is considered. This again disfavors the low-energy bandtail states, as otherwise the thermal filling should be more likely for the low-energy (here standing-) PL component. (iii) The moving-PL component corresponds to a stronger electron-phonon interaction than the corresponding standing-PL. The annealing slightly enhances the interaction for the InGaAs SQW but not for the InGaNAs SQW. For the both SQWs, the annealed samples show a similar strength of the interaction, hinting the

TABLE IV. Parameters of Eq. (3) for fitting the standing- and moving- $P L$ line widths for as-grown and annealed InGaAs (A) and InGaNAs (B) SQWs. $\Gamma_{i}^{0}$ and $\beta_{i}$ in unit of meV, and $i$ for standing (s) and moving (m) components.

\begin{tabular}{lcccccc}
\hline \hline Sample & A-ag & A-600 & A-750 & B-ag & B-600 & B-750 \\
\hline$\Gamma_{\mathrm{s}}^{0}$ & 11.0 & 14.3 & 10.5 & 20.8 & 17.3 & 12.2 \\
$\beta_{\mathrm{s}}$ & 22.2 & 31.3 & 20.8 & 26.0 & 28.2 & 17.6 \\
$\Gamma_{\mathrm{m}}^{0}$ & 21.1 & 26.2 & 21.5 & 35.7 & 31.5 & 21.4 \\
$\beta_{\mathrm{m}}$ & 76.6 & 114.7 & 102.8 & 102.9 & 105.7 & 104.8 \\
\hline \hline
\end{tabular}


interaction to be in a different region of the QW layer for the standing- and moving-PL components, respectively.

\section{Discussion}

To well understand the experimental observations, neither the change of interlayer crystal quality nor the interdiffusion effects could be sufficient. Instead, a joint effect of intra-layer crystal quality, interfacial fluctuation of the quantum well layer, and interfacial diffusion has to be considered: (i) the InGaAs QW layer manifests fluctuation of In composition near the GaAs/InGaAs interface due to nonuniform In segregation, ${ }^{17}$ which can be described equivalently with a part of standard-thickness QW plus a part of slightly narrower QW, the latter leads to higher energy states for high temperature/excitation, and correspondingly the moving- $P L$ component on the higher-energy side of the standing- $P L$ component; (ii) incorporation of $\mathrm{N}$ in InGaAs degrades the intra-layer crystal quality and deteriorates the interfacial fluctuation, ${ }^{15}$ the former broadens the linewidth of each of the PL components, while the latter provides also lower energy states at low temperature/excitation and hence a cross-over of the standing- and moving-PL components at a medium excitation, and tends to enhance the separation of the two PL components at high excitation; and (iii) annealing at proper temperature improves the intra-layer crystal quality $^{17}$ and drives interfacial In-Ga interdiffusion ${ }^{30}$ along the vertical and horizontal directions, the former narrows the linewidth, while the latter weakens the In segregation, smooths the interfacial fluctuation, shrinks the effective QWlayer thickness, and hence leads to the blueshift and narrowing the separation of the two PL components.

To check if the interdiffusion-induced narrowing of the QW layer is qualified for the blueshift, the ground-state transition energy is calculated with a square-well assumption. ${ }^{43}$ The band offset is determined by the model-solid method, ${ }^{34,35}$ and the dilute- $\mathrm{N}$ effect is estimated with the two-level repulsion model. ${ }^{44,45}$ Figure 10 depicts the transition energy of $\mathrm{In}_{0.375} \mathrm{Ga}_{0.625} \mathrm{As} / \mathrm{GaAs}$ and $\operatorname{In}_{0.375} \mathrm{Ga}_{0.625} \mathrm{~N}_{0.012} \mathrm{As}_{0.988} / \mathrm{GaAs}$ SQWs with the transition energy of 7-nm-thick $\operatorname{In}_{0.375} \mathrm{Ga}_{0.625} \mathrm{As} /$ GaAs SQW as reference.

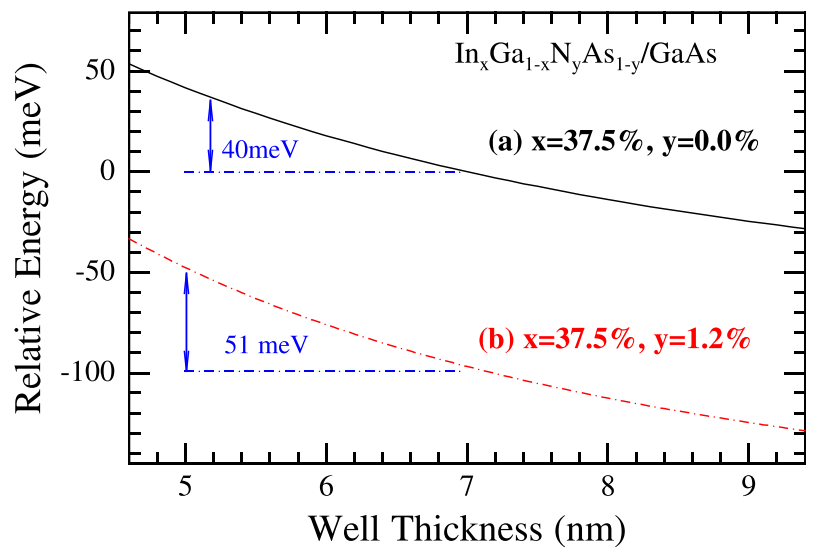

FIG. 10. Relative transition energy as a function of QW-layer thickness for $\mathrm{In}_{x} \mathrm{Ga}_{1-x} \mathrm{~N}_{y} \mathrm{As}_{1-y} / \mathrm{GaAs} \mathrm{SQW}$ by model-solid method and two-level repulsion model, 7-nm-thick $\mathrm{In}_{0.375} \mathrm{Ga}_{0.625} \mathrm{As} / \mathrm{GaAs} \mathrm{SQW}$ as reference energy.
From the energy difference between the as-grown and annealed samples, the equivalent thickness of the InGaAs and InGaNAs QW layers should shrink, respectively, to about $6.6 \mathrm{~nm}$ and $6.2 \mathrm{~nm}$ for the $600^{\circ} \mathrm{C}$, and $5.1 \mathrm{~nm}$ and $5.0 \mathrm{~nm}$ for the $750^{\circ} \mathrm{C}$ annealed samples. Accordingly, the differences to the equivalent narrower $\mathrm{QW}$ parts of the InGaAs and InGaNAs SQWs are, respectively, 0.5 and $0.7 \mathrm{~nm}$ for the as-grown, 0.4 and $0.8 \mathrm{~nm}$ for the $600^{\circ} \mathrm{C}$, and $0.3 \mathrm{~nm}$ for the $750^{\circ} \mathrm{C}$ annealed samples.

Such amount of shrinkage is reasonable with respect to the previous studies. Liu et al. studied annealing effect on 8nm-thick $\mathrm{In}_{0.372} \mathrm{Ga}_{0.628} \mathrm{~N}_{0.015} \mathrm{As}_{0.985} / \mathrm{GaAs} \mathrm{SQW}$ and concluded that annealing-induced interdiffusion length of $\leq 2 \mathrm{~nm}$ corresponds to PL peak blueshift of $18-28 \mathrm{meV} .{ }^{25}$ Bouragba et al. indicated that while a nitrogen free $\mathrm{InGaAs} / \mathrm{GaAs} \mathrm{SQW}$ sample shows the In-Ga interdiffusion, the introduction of nitrogen moderately enhances the phenomenon. ${ }^{30}$ Shirakata et al. indicated that for 10-nm-thick $\operatorname{In}_{0.32} \mathrm{Ga}_{0.68} \mathrm{~N}_{0.01} \mathrm{As}_{0.99} /$ GaAs SQW, annealing improves the crystal quality to be comparable with that of corresponding $\mathrm{N}$-free sample. ${ }^{24}$ Lai et al. showed for 6-nm-thick $\mathrm{In}_{0.34} \mathrm{Ga}_{0.66} \mathrm{~N}_{x} \mathrm{As}_{1-x} / \mathrm{GaAs}$ SQWs, the PL peak energy manifests a pronounced temperaturedependent $\mathrm{S}$-shaped for the dilute-N samples at low temperatures, ${ }^{22}$ while it follows the empirical Varshni model in the high-temperature region with significant reduction in the temperature dependence as compared to the $\mathrm{N}$-free sample, the latter manifests similar $\alpha$ and $\beta$ values as those of the main PL peaks for the A-ag and B-600/B-750 as listed in Table III.

It is noteworthy that (i) a study of RTA effects on InGaNAs/GaAs SQWs correlated the annealing-induced blueshift to the $\mathrm{N}$ content in the QW layer but not the In interdiffusion, ${ }^{23}$ which seems to be contrary to the above discussion. Note, however, that the reference $\mathrm{N}$-free sample manifested significantly smaller blueshift, which is not the case in the present study. (ii) For a similar narrowing of the QW layer, while 40 and $51 \mathrm{meV}$ blueshift is predicted for 7-nm-thick InGaAs and InGaNAs SQWs, respectively, much reduced values of 24 and $27 \mathrm{meV}$ are expected if the QW layer is $9 \mathrm{~nm}$, which are very closed to the previous reports. ${ }^{23-25}$ (iii) A density-dependent time-resolved PL study of a $8 \mathrm{~nm}$ InGaAs/ GaAs SQW derived an exciton binding energy of about $6.5 \mathrm{meV},{ }^{26}$ and a lineshape analysis-assisted temperature-dependent PL study of $6 \mathrm{~nm} \mathrm{In}_{0.4} \mathrm{Ga}_{0.6} \mathrm{As} / \mathrm{GaAs}$ deduced an exciton binding energy of about $9.7 \mathrm{meV},{ }^{27}$ both are very similar to those as listed in Table II. Nevertheless, a drastic enhancement of excitonic effect by dilute nitrogen was suggested and a very low $\mathrm{N}$ content of about $0.5 \%$ was considered to increase surprisingly the exciton binding energy in the $6 \mathrm{~nm}$ $\mathrm{In}_{0.4} \mathrm{Ga}_{0.6} \mathrm{~N}_{0.005} \mathrm{As}_{0.995} / \mathrm{GaAs}$ by $80 \%$ relative to the referential InGaAs/GaAs SQW to $17.5 \mathrm{meV},{ }^{27}$ which is obviously inconsistent with those as listed in Table II. Note, however, that the lineshape analysis was based on a two-component assumption of additive of excitonic and free-carrier transitions and the exciton binding energy was derived indirectly with a plot of the ratio of free carriers to exciton populations against $1 / k_{B} T{ }^{27}$ this is, however, in clear contrast to the nature of two excitonic components and the direct derivation of the exciton binding energy by diamagnetic shift in this study. (iv) As the QW interdiffusion has been applied as a post-growth 
approach for engineering the bandgap on selected areas to achieve monolithic integration of different optoelectronic functions, ${ }^{46,47}$ the effect of the interfacial fluctuation of either thickness or composition deserves special attention in the annealing-induced bandshift engineering.

\section{CONCLUSION}

To conclude, for the $\operatorname{InGa}(\mathrm{N}) \mathrm{As} / \mathrm{GaAs}$ SQWs not showing $S$-shape evolution of PL-peak energy with temperature, the PL spectra at different excitation powers, temperatures and magnetic fields can be well fitted by a two-PL-component assumption, and the PL components are both excitonic and of interband ground-state related transition in the QW portions with slightly different well thicknesses. The RTA introduces similar levels of blueshift and narrowing to the PL components of the both $\operatorname{InGa}(\mathrm{N}) \mathrm{As}$ SQWs with and without $\mathrm{N}$ content, and the mechanisms behind the annealing effects are inferred that (i) the In segregation near the interface causes less-In QW region, which corresponds equivalently to higher-energy states and hence the high-energy moving- $P L$ component, $\mathrm{N}$ introduces additional fluctuation to the interface and provides also lowerenergy states, as shown by the excitation power-dependent $\mathrm{PL}$ analysis in Sec. III A; (ii) the annealing improves the intra-layer crystal quality and hence narrows the PL components, as indicated by the excitation power-dependent PL analysis in Sec. III A and the temperature-dependent PL analysis in Sec. III C. It also enhances the interfacial In-Ga interdiffusion and leads to an equivalent reduction of the QW thickness and interface fluctuation, and hence the blueshift of the PL components and reduction of the PLcomponents' separation as revealed by the temperaturedependent PL analysis in Sec. III C; and (iii) the interfacial In-Ga interdiffusion does not change obviously by the small amount $\mathrm{N}$ content (here is about 1.2\%) and hence similar levels of PL-component narrowing and blueshift are observed after a nominally identical RTA treatment. The introduction of $1.2 \%$ nitrogen into InGaAs increases the exciton binding energy by a moderate level of about $15 \%$ for the $750^{\circ} \mathrm{C}$ annealing as derived by the magneto-PL analysis in Sec. III B.

As for neither of the $\operatorname{InGa}(\mathrm{N})$ As SQWs does the bandtail explicitly show up even at rather low excitation power/temperature, the as-grown SQWs do already possess very good optical quality and hence the interfacial effects play an important role in the evolution of the PL lineshape with excitation, temperature, and magnetic field. From this viewpoint, PL measurements with sufficient spectral resolution and SNR together with lineshape analysis will be desirable and informative for probing the interfacial modification by annealing.

\section{ACKNOWLEDGMENTS}

The work was supported by the MOST 973 Program (2014CB643901 and 2013CB632805), the STCSM (11JC1413800), and the NSFC $(61176075,11274329$, and 61290302) of China.
${ }^{1}$ J. Shao, R. Winterhoff, A. Dörnen, E. Baars, and J. Chu, Phys. Rev. B 68 , 165327 (2003).

${ }^{2}$ J. Shao, W. Lu, X. Lü, F. Yue, Z. Li, S. Guo, and J. Chu, Rev. Sci. Instrum. 77, 063104 (2006).

${ }^{3}$ Y. P. Varshni, Physica 34, 149 (1967).

${ }^{4}$ K. P. O'Donnell and X. Chen, Appl. Phys. Lett. 58, 2924 (1991).

${ }^{5}$ G. Ortner, M. Schwab, M. Bayer, R. Pässler, S. Fafard, Z. Wasilewski, P. Hawrylak, and A. Forchel, Phys. Rev. B 72, 085328 (2005).

${ }^{6}$ M. Hugues, B. Damilano, J.-Y. Duboz, and J. Massies, Phys. Rev. B 75, 115337 (2007).

${ }^{7}$ J. Shao, X. Lü, F. Yue, W. Huang, S. Guo, and J. Chu, J. Appl. Phys. 100, 053522 (2006).

${ }^{8}$ J. Shao, L. Chen, W. Lu, X. Lu, L. Zhu, S. Guo, L. He, and J. Chu, Appl. Phys. Lett. 96, 121915 (2010).

${ }^{9}$ X. Zhang, J. Shao, L. Chen, X. Lü, S. Guo, L. He, and J. Chu, J. Appl. Phys. 110, 043503 (2011)

${ }^{10}$ X. Chen, Y. Song, L. Zhu, S. M. Wang, W. Lu, S. Guo, and J. Shao, J. Appl. Phys. 113, 153505 (2013).

${ }^{11}$ J. N. Baillargeon, K. Y. Cheng, G. E. Hofler, P. J. Pearah, and K. C. Hsieh, Appl. Phys. Lett. 60, 2540 (1992).

${ }^{12}$ M. Kondow, K. Uomi, A. Niwa, T. Kitatani, S. Watahiki, and Y. Yazawa, Jpn. J. Appl. Phys. Part 1 35, 1273 (1996).

${ }^{13}$ N. Tansu, J.-Y. Yeh, and L. J. Mawst, Appl. Phys. Lett. 83, 2512 (2003).

${ }^{14}$ X. Wang, S. M. Wang, Y. Q. Wei, M. Sadeghi, and A. Larsson, Electron. Lett. 40, 1338 (2004).

${ }^{15}$ M. M. Oye, T. J. Mattord, G. A. Hallock, S. R. Bank, M. A. Wistey, J. M. Reifsnider, A. J. Ptak, H. B. Yuen, J. S. Harris, and A. L. Holmes, Appl. Phys. Lett. 91, 191903 (2007).

${ }^{16}$ I. A. Buyanova, W. M. Chen, G. Pozina, J. P. Bergman, B. Monemar, H. P. Xin, and C. W. Tu, Appl. Phys. Lett. 75, 501 (1999).

${ }^{17}$ H. Zhao, S. M. Wang, Q. X. Zhao, Z. H. Lai, M. Sadeghi, and A. Larsson, Semicond. Sci. Technol. 23, 125002 (2008).

${ }^{18}$ J. C. P. Chang, J. M. Woodall, M. R. Melloch, I. Lahiri, D. D. Nolte, N. Y. Li, and C. W. Tu, Appl. Phys. Lett. 67, 3491 (1995).

${ }^{19}$ Y. Kajikawa, N. Nishimoto, D. Fujioka, and K. Ichida, Jpn. J. Appl. Phys. Part 1 45, 2412 (2006).

${ }^{20}$ H. Zhao, Y. Q. Xu, H. Q. Ni, S. Y. Zhang, D. H. Wu, Q. Han, R. H. Wu, and Z. C. Niu, J. Appl. Phys. 99, 034903 (2006).

${ }^{21}$ A. Matsumoto, A. Matsushita, Y. Takei, K. Akahane, Y. Matsushima, H. Ishikawa, and K. Utaka, Appl. Phys. Express 7, 092801 (2014).

${ }^{22}$ F.-I. Lai, S. Y. Kuo, J. S. Wang, H. C. Kuo, S. C. Wang, H. S. Wang, C. T. Liang, and Y. F. Chen, J. Vac. Sci. Technol. A 24, 1223 (2006).

${ }^{23}$ V. Liverini, A. Rutz, U. Keller, and S. Schön, J. Appl. Phys. 99, 113103 (2006).

${ }^{24}$ S. Shirakata, M. Kondow, and T. Kitatani, Appl. Phys. Lett. 80, 2087 (2002).

${ }^{25}$ H. F. Liu, V. Dixit, and N. Xiang, J. Appl. Phys. 99, 013503 (2006).

${ }^{26}$ L. Kappei, J. Szczytko, F. Morier-Genoud, and B. Deveaud, Phys. Rev. Lett. 94, 147403 (2005).

${ }^{27}$ L. Xu, D. Patel, C. S. Menoni, J. Y. Yeh, L. J. Mawst, and N. Tansu, Appl. Phys. Lett. 89, 171112 (2006).

${ }^{28}$ L. Zhu, J. Shao, X. Lü, S. Guo, and J. Chu, J. Appl. Phys. 109, 013509 (2011).

${ }^{29}$ J. Shao, D. Haase, A. Dörnen, V. Härle, and F. Scholz, J. Appl. Phys. 87, 4303 (2000).

${ }^{30}$ T. Bouragba, M. Mihailovic, F. Reveret, P. Disseix, J. Leymarie, A. Vasson, B. Damilano, M. Hugues, J. Massies, and J. Y. Duboz, J. Appl. Phys. 101, 073510 (2007).

${ }^{31}$ M. Sugawara, Phys. Rev. B 45, 11423 (1992).

${ }^{32}$ I. Aksenov, J. Kusano, Y. Aoyagi, T. Sugano, T. Yasuda, and Y. Segawa, Phys. Rev. B 51, 4278 (1995).

${ }^{33}$ C. Klingshirn, Semiconductor Optics, 2nd ed. (Springer-Verlag, Berlin Heidelberg, 2005).

${ }^{34}$ C. G. V. de Walle, Phys. Rev. B 39, 1871 (1989).

${ }^{35}$ J. Shao, A. Dörnen, R. Winterhoff, and F. Scholz, Phys. Rev. B 66, 035109 (2002).

${ }^{36}$ I. Vurgaftman and J. R. Meyer, J. Appl. Phys. 94, 3675 (2003).

${ }^{37}$ J. Humlíček, E. Schmidt, L. Bočánek, R. Švehla, and K. Ploog, Phys. Rev. B 48, 5241 (1993).

${ }^{38}$ J. Shao, W. Lu, G. K. O. Tsen, S. Guo, and J. M. Dell, J. Appl. Phys. 112, 063512 (2012).

${ }^{39}$ P. Lautenschlager, M. Garriga, L. Vina, and M. Cardona, Phys. Rev. B 36, 4821 (1987)

${ }^{40}$ H. Qiao, K. A. Abel, F. C. J. M. van Veggel, and J. F. Young, Phys. Rev. B 82, 165435 (2010). 
${ }^{41}$ R. Heitz, M. Grundmann, N. N. Ledentsov, L. Eckey, M. Veit, D. Bimberg, V. M. Ustinov, A. Y. Egorov, A. E. Zhukov, P. S. Kopçev, and Z. I. Alferov, Appl. Phys. Lett. 68, 361 (1996).

${ }^{42}$ C. Bungaro, K. Rapcewicz, and J. Bernholc, Phys. Rev. B 61, 6720 (2000).

${ }^{43}$ J. Shao, A. Dörnen, E. Baars, V. Härle, F. Scholz, S. Guo, and J. Chu, J. Appl. Phys. 93, 951 (2003).
${ }^{44}$ W. Shan, W. Walukiewicz, J. W. Ager III, E. E. Haller, J. F. Geisz, D. J. Friedman, J. M. Olson, and S. R. Kurtz, Phys. Rev. Lett. 82, 1221 (1999).

${ }^{45}$ J. Shao, W. Lu, M. Sadeghi, X. Lü, S. M. Wang, L. Ma, and A. Larsson, Appl. Phys. Lett. 93, 031904 (2008).

${ }^{46}$ K.-H. Lee, K. Thomas, A. Gocalinska, M. Manganaro, E. Pelucchi, F. H. Peters, and B. Corbett, J. Appl. Phys. 112, 093109 (2012).

${ }^{47}$ R. Beal, V. Aimez, and J. J. Dubowski, Opt. Express 23, 1073 (2015). 